\title{
Germanica
}

\section{Du mal d'être au non-être : le Barabbas de Pär}

Lagerkvist

Vom schmerzvollen Dasein zum Nicht-Sein: Pär Lagerkvists Barabbas

\section{Georges Ueberschlag}

\section{OpenEdition}

1 Journals

Édition électronique

URL : http://journals.openedition.org/germanica/2631

DOI : 10.4000/germanica.2631

ISSN : 2107-0784

Éditeur

Université de Lille

\section{Édition imprimée}

Date de publication : 31 décembre 1989

Pagination : 123-143

ISSN : 0984-2632

\section{Référence électronique}

Georges Ueberschlag, « Du mal d'être au non-être : le Barabbas de Pär Lagerkvist », Germanica [En ligne], 5 | 1989, mis en ligne le 09 février 2015, consulté le 06 octobre 2020. URL : http:// journals.openedition.org/germanica/2631 ; DOI : https://doi.org/10.4000/germanica.2631

Ce document a été généré automatiquement le 6 octobre 2020.

(C) Tous droits réservés 


\section{Du mal d'être au non-être : le Barabbas de Pär Lagerkvist}

Vom schmerzvollen Dasein zum Nicht-Sein: Pär Lagerkvists Barabbas

\section{Georges Ueberschlag}

1 Barabbas - un simple nom dans les Écritures, personnage à la périphérie et qui reste dans l'ombre.

2 Son existence est bien attestée par les Évangiles. Marc et Luc le présentent comme un « prisonnier fameux » incarcéré avec des complices pour un meurtre commis au cours d'une sédition, alors que Jean parle simplement de lui comme d'un brigand. Mais il n'a pas de contours précis, pas de véritable dimension individuelle.

3 Comme tout le monde le sait, il est celui que Pilate libéra à la place de Jésus de Nazareth. Mais tout aussitôt après il disparait de la scène. Pour devenir quoi ?

4 C'est la question que Pär Lagerkvist se pose dans son roman Barabbas paru en 1950. Elle en sera le point de départ, et le point d'arrivée. Que peut signifier pour un individu le fait que quelqu'un d'autre meurt à sa place, pour que lui-même vive et soit libre?

5 Si le Christ est mort pour nous, comme le proclame la foi chrétienne, combien plus cette affirmation est-elle vérité pour Barabbas, réalité tangible. Un autre est allé mourir à sa place. Il est sauvé à la vie par la condamnation à mort de Jésus. «Crucifie-le et livre nous Barabbas » avait crié la foule excitée des Juifs.

6 Mais le salut inespéré, immérité aussi, dont les raisons profondes lui échappent, rend Barabbas à une existence dont il avait sans doute déjà fait son deuil, le rejette dans une vie et dans une liberté dont il ne sait plus quoi faire, et qui deviennent un véritable cadeau empoisonné. Il y emporte ce qu'il n'avait probablement jamais encore connu, la mauvaise conscience et le mal d'être, un mal d'être qui l'entraînera jusqu'au bord du gouffre existentiel, du néant, du non-être.

7 Certes, des personnages de la Passion du Christ avaient déjà tenté auparavant d'autres écrivains suédois. 
En 1886, Tor Hedberg avait publié un récit intitulé Judas, une histoire de la Passion, où il insiste sur l'inéluctable nécessité de la trahison et du mal, pour que le bien advienne. Et en 1928 Hjalmar Söderberg, dont l'athéisme avait déjà fait quelques vagues en Suède, tenta dans une sorte d'essai, Jésus - Barabbas, d'identifier les deux personnages, affirmant qu'il ne s'agissait que d'une seule et même personne. Il s'appuyait, ce faisant, sur certains manuscrits de l'évangile de Matthieu qui disaient que Barabbas avait le même "prénom » que le Maître. Mais sa tentative, plus encore que celle que Ernest Renan avait entreprise dans sa Vie de Jésus dans le même sens, tourna court ${ }^{1}$.

Ce ne sont donc certainement pas ces exemples qui ont pu inciter Pär Lagerkvist à scruter, à imaginer le destin de Barabbas après la Passion. L'environnement biblique lui avait été familier depuis son enfance, et il avait entrepris en 1934 un voyage en Grèce et en Palestine qui le confirmait dans sa familiarité avec la Terre Sainte ${ }^{2}$.

10 Pour qui connaît la trajectoire spirituelle, philosophique de Pär Lagerkvist ${ }^{3}$, il ne saurait guère être surprenant de voir que l'auteur se soit emparé de ce personnage de Barabbas pour creuser sa vision des relations entre l'homme et le divin, entre le monde et le surnaturel. S'appuyant sur le récit de Saint Jean, dont l'évangile d'amour sera parmi ce qui le fascinera - et le paralysera - le plus dans le message chrétien, Pär Lagerkvist considère Barabbas simplement au départ comme un brigand, comme l'avait d'ailleurs déjà fait François Mauriac, lui aussi, dans sa Vie de Jésus.

11 Mais pour Lagerkvist il deviendra Barabbas le libéré - Barabbas den frikände, et avec cette épithète qui s'accroche à lui comme une malédiction, toute la problématique humaine du mal d'être est posée.

\section{I.}

Le roman de Barabbas, pour lequel Pär Lagerkvist avait obtenu en 1951 le Prix Nobel de littérature, est le premier livre où l'auteur affronte vraiment le thème central de la foi chrétienne, celui de la rédemption par la mort du Christ. Au centre de l'action se trouve un homme dans l'âme duquel l'appel de la lumière et les forces négatives des ténèbres se livre une âpre lutte, jusqu'à cette issue fatale où le héros se trouve cloué sur une croix, comme jadis le Christ lui-même.

Pour comprendre la manière dont Pär Lagerkvist aborde dans ce roman les « questions essentielles ", incitant le lecteur à le faire avec lui, une manière ambiguë comme si souvent chez lui, il n'est sans doute pas inutile de jeter un coup d'œil sur son évolution, son itinéraire.

14 Pär Lagerkvist est né en 1891 dans le Småland, une région boisée assez fermée et assez pauvre, dont la paysannerie restait fermement attachée à la tradition. On y vivait généralement replié sur soi-même. La religion imprégnait tout le climat, à la ville comme à la campagne, et conditionnait toute discussion d'idées. L'Église officielle y était très conservatrice, et hostile à tous les mouvements populaires, le libéralisme aussi bien que le socialisme, les ligues de tempérance ou les sectes religieuses.

Le milieu familial du jeune Pär était fortement marqué par le piétisme ${ }^{4}$, par la lecture de la Bible, l'acceptation joyeuse de la volonté de Dieu et la pensée constante de la mort. Il y régnait, comme l'écrira l'auteur, une atmosphère « de paix, de chaleur et de bonté ». C'était un milieu protégé de l'extérieur, où la réponse aux "questions essentielles » était déjà toute donnée. 
Si le mal d'être inhérent à toute existence humaine trouvait à s'y manifester, il était tout au plus une conséquence de la pauvreté. Mais il n'était guère, ou même jamais, d'ordre métaphysique et religieux.

L'exemple du grand-père a particulièrement impressionné le jeune Pär Lagerkvist, sa foi et sa façon résignée et joyeuse de vivre. Il s'en souviendra constamment plus tard, dans sa quête individuelle de Dieu et de la religion.

L'influence du milieu familial a, en effet, largement conditionné les futurs conflits intérieurs de l'auteur. Toute son œuvre en est l'écho. Conflits inévitables, car en dehors de cet enclos protégé qu'était la famille, les idées bouillonnaient alors, même dans le Småland.

9 Pär Lagerkvist, jeune lycéen avide de connaissances, s'initia tôt aux nouveaux courants philosophiques, au déterminisme et au radicalisme religieux, au darwinisme «qui balaya Dieu et toute espérance ${ }^{5}$ ». C'était entamer la longue lutte entre la foi et le doute - que le plus fort gagne ! - et le chemin d'une religion (religere !) conquise en individu libre, si conquête il devait y avoir.

« Les savants ont découvert un tas de choses, note-t-il à ce sujet..., mais au-delà de leurs limites qu'y a-t-il ? $^{\prime}$ » Interrogation fondamentale qui traverse toute l'œuvre de l'auteur.

21 D'autant plus que Pär Lagerkvist, ne pouvant jamais se dégager complètement de son héritage spirituel, ou ne le voulant pas, ni assumer pleinement, d'autre part, les changements idéologiques subséquents à l'évolution économique et sociale, ou parfois aussi les anticipant, s'enfoncera dans une angoisse existentielle tragique, celle d'un homme qui a besoin d'un ancrage solide et qui ne le trouve pas.

2 Le jeune Pär, ne pouvant plus prier avec ses parents, ne s'était-il pas trouvé un jour dans la forêt une "pierre de la prière" à laquelle il allait confier toutes ses préoccupations intimes!

23 L'inquiétude intérieure était donc déjà devenue sa compagne, lui donnant l'impression qu'il n'était plus chez lui nulle part, qu'il n'était plus qu'un étranger dans ce monde, un simple hôte - gäst hos verkligheten.

24 Étudiant à Upsal, il suit le sillage de la revue radicale socialiste Stormklockan. S'initiant aux mouvements littéraires européens, à l'expressionnisme surtout, et entamant presque aussitôt sa carrière d'écrivain, la seule qui lui semblait possible, il publia dès 1913 ses écrits avant-gardistes, dont Ordkonst och bildkonst (Écritures et arts plastiques) qui se présente comme une synthèse habile du credo cubiste français, du futurisme italien et de l'expressionnisme allemand. Dans son analyse il souligne fréquemment combien tout art est intimement lié au religieux, tributaire des sentiments humains élémentaires comme l'amour, la haine, l'angoisse, le doute.

25 Trois ans plus tard paraît son premier recueil de poèmes Ångest (Angoissé), dans lequel déjà presque tout est dit.

"L'angoisse, l'angoisse est mon héritage », tel en est le leitmotiv. Et ce thème va se retrouver, avec la fidélité du " pauvre enfant vêtu de noir » de la Nuit de décembre de Musset, dans presque toutes les œuvres. Avec la vague de la littérature existentialiste qui déferlera sur l'Europe après 1945, il va connaître un succès croissant et contribuer fortement à la renommée de Lagerkvist. 
27 Le pessimisme dostoïevskien qu'on y perçoit, un pessimisme sans compromis, n'arrivera cependant jamais chez Pär Lagerkvist à faire oublier totalement la vision idéaliste de l'homme qui se bat contre le mal. Cette vision affleure toujours comme un filet d'eau claire au milieu d'un fleuve d'eaux boueuses.

Ces deux pôles, points forts et permanents de toute Weltanschauung, et causes de conflits insolubles, vont alimenter la pensée de Lagerkvist, sa vision de l'homme et de Dieu exprimée à travers le symbolisme de ses œuvres ${ }^{7}$.

29 L'écrivain s'y affirme toujours davantage comme le prophète du religieux. La lutte contre le mal et ses manifestations immédiates, qu'il s'agisse du totalitarisme ou du mal de vivre d'une humanité déboussolée, s'impose à lui comme une tâche impérative.

La philosophie de Kierkegaard, qu'il relira vers les années 1950 avec un intérêt renouvelé ${ }^{8}$, l'amène à formuler de façon plus précise sa position personnelle face à ce problème du mal, lié à celui, plus central encore, de la foi.

31 N'adhérant à aucune religion, les considérant toutes comme des manifestations passagères, Lagerkvist croit, en revanche, en une source de la dimension transcendantale de l'homme, une source qu'il ne sait situer, qu'il ne sait définir, échappant à tout entendement humain, une Puissance. Et il résume sa confession dans cette formule, lucide et lapidaire : «Jar är en troende utan tro, en religiös ateist ${ }^{9}$ » (Je suis un croyant sans croyance, un athée religieux).

32 Barabbas est l'expression directe de cette quête religieuse, plus fortement encore que les autres œuvres. Une quête angoissée qui se situe dans un monde à la dérive, un monde qui répète que Dieu est mort, comme pour se donner l'illusion de dominer l'absurde. Et qui pourtant ne cesse de lutter avec lui - si ce n'est contre lui, gigantesque combat de Jacob contre l'Ange. Une quête nourrie par l'inquiétude, dont le thème sera traité de façon plus imagée, plus symbolique dans les dernières œuvres de Lagérkvist, notamment dans le cycle de poèmes Aftonland (Pays du couchant), 1953. Mais une quête qui toujours suit les méandres de l'attitude ambiguë de Lagerkvist face au divin, faite à la fois d'attirance ouverte, de bonne volonté et de refus malgré lui. Une quête qui ne laissera jamais l'homme en paix, car jamais le bienêtre ne sera d'ordre religieux ou métaphysique. Le mal d'être toujours.

II.

33 La lutte avec Dieu est décrite, dans Barabbas, dans le cadre historique de l'existence terrestre de Jésus de Nazareth. Celle-ci n'en fournit que le point de départ, mais elle contraint le héros, comme elle contraint l'auteur, et tout homme, à se prononcer : « Et vous, qui dites-vous que je suis ? $^{10}$ »

34 Le matériel historique est employé par Lagerkvist avec une totale liberté poétique, la liberté du créateur. Bien que tout démontre sa très bonne connaissance du Nouveau Testament, sa familiarité avec le milieu dans lequel il place son roman, il ne cherche pas à nous donner une reconstitution historique. Les premiers chrétiens, par exemple, ne se sont pas comportés comme les décrit Pär Lagerkvist. Celui-ci ne se soucie pas de détails, il procède par projection et projette donc des manifestations de vie chrétienne qu'il a connues dans sa jeunesse, celles des sectes religieuses, dans le monde des chrétiens primitifs, un monde de pauvreté et de simples d'esprits, qu'il nous présente, du reste, sans aucune intention polémique. 
Si Lagerkvist situe son action dans une sorte de Jérusalem du Småland, c'est que la vérité historique, ce n'est pas son problème. Et ce n'est surtout pas l'essentiel.

"Les apparences d'un personnage, cela ne signifie pas grand-chose ${ }^{11}$ ", écrit-il dès les premières pages du roman.

À côté de Barabbas et de Jésus - un homme chétif, bizarre, qui n'a pas de poils sur la poitrine, note le premier, et dont émane pourtant une force et un attrait indéfinissables - il y a d'autres personnages historiques comme Pierre et Marie, la mère de Jésus, Marie-Madeleine, une pauvre fille sans charme, une réprouvée que Lagerkvist nous présente simplement sous un sobriquet, le Bec-de-lièvre.

Ce sont des hommes et des femmes rudes, à la manière des paysans du Småland. Pierre est un grand gaillard naïf aux yeux bleus, et l'auteur insiste à plusieurs reprises sur les allures paysannes de Marie. Ils sont décrit selon les idées reçues et bien ancrées dans la tradition piétiste. Ils ne sont pas du tout construits selon une étude approfondie des travaux de l'exégèse biblique moderne.

Le milieu des premiers chrétiens est présenté dans des couleurs assez sombres. Ceux-ci évoluent dans les bas-fonds de la société, dans les arrières-boutiques ou parmi le rebut de la vallée des lépreux. Ils font partie des classes sociales les plus déshéritées, selon une idée qui s'était répandue au Siècle des Lumières ${ }^{12}$, que Voltaire, entre autres, s'était appropriée, et qui voulait que le christianisme ait pris naissance dans les basses couches de la population ${ }^{13}$.

Mais cela ne veut pas dire que Lagerkvist ait pris à son compte les critiques de Nietzsche, par exemple, concernant les origines plébéiennes du christianisme. Même s'il s'inspire de cette idée, il sait aussi que l'exégèse biblique du début du $\mathrm{XX}^{\mathrm{e}}$ siècle, a montré que les premiers chrétiens ne se recrutaient pas uniquement parmi les plus démunis, parmi ceux qui avaient besoin d'être renvoyés à la consolation de la venue d'un autre Royaume.

41 Cependant, si Lagerkvist situe son roman et ses personnages dans ce milieu - et Barabbas en est un représentant sans aucune concession - c'est que ceux-ci prennent valeur de symbole pour le mal d'être des hommes d'aujourd'hui, des hommes de toujours. Ils ne pouvaient donc, psychologiquement, guère se situer ailleurs. L'auteur a senti avec une grande justesse et situé de façon significative son sujet.

Son sujet... et son personnage.

Barabbas donc, un nom auquel l'auteur veut donner vie et dont il ne fait pourtant pas un individu avec son autonomie propre, malgré les traits bien particuliers dont il le dote, physiquement et psychologiquement, nous est décrit comme un rouquin à la poitrine velue, les yeux profondément enfoncés dans leur orbite, le visage mangé par une barbe rousse et par une longue balafre sur la joue.

C'est un costaud assez fruste, faisant volontiers étalage et usage de sa force physique, ou plutôt l'ayant fait jadis.

Car soudain il se voit en proie à des mouvements de l'âme qu'il ne sait plus contrôler.

C'est là que le prend Lagerkvist pour en faire le type du personnage enfermé en luimême, qui se débat au creux de la condition humaine, dans un huis clos sartrien, et dont trois étapes jalonnent le parcours.

Après la séance devant le tribunal de Pilate, le voilà donc libre. Barabbas le libéré - den frikände. 
Et où le conduisent ses premiers pas d'homme libéré ?

À l'écart, caché derrière les fourrés, il assiste à la crucifixion de l'Autre, qu'entoure, lui semble-t-il, un étrange halo. Et il voit les événements qui se produisent, les ténèbres qui se font subitement et qui le laissent perplexe.

Puis il retourne dans son milieu coutumier, celui des prostituées de Jérusalem qui l'accueillent avec ravissement. Quelle chance il a eue! Elles veulent le toucher comme on toucherait la corde d'un pendu miraculé. Mais il se montre curieusement taciturne, devenu étranger à tout et à tous, à son passé surtout ${ }^{14}$.

Errant dans Jérusalem, il rencontre des disciples qui lui parlent de ce qui doit se produire à l'aube du troisième jour, selon la parole du Maître.

Il retourne donc au tombeau, sans bien savoir pourquoi, et s'aperçoit que soudain, au premier rayon du soleil du matin qui est venu le frapper et qui a jailli comme un éclair, celui-ci est vide. Barabbas voudrait alors bien croire au miracle, comme le Bec-de-lièvre présent aussi sur les lieux, au miracle annoncé par les disciples. Mais la méfiance, la peur d'avoir été simplement victime d'hallucinations sont plus fortes.

Ruminant tout ce qu'il vient de vivre, fuyant la compagnie des hommes, il ne peut cependant éviter - ou le recherche-t-il obscurément ? - de tomber sur des partisans de ce rabbi crucifié, autour de la communauté desquels il rôde parfois comme un loup solitaire. Ceux-ci mettent en pratique la loi d'amour - älsken varandra - et veulent initier le nouveau venu à leur croyance.

Devant son scepticisme ils lui proposent même d'aller voir de ses propres yeux un ressuscité, Lazare. Quelqu'un qui doit connaître donc la vérité sur ce royaume des ténèbres, dont lui, et l'Autre, sont revenus. Le royaume des ténèbres, la mort, lui répond Lazare comme à contre-cœur, que veux-tu que je t'en dise?

«Le royaume de la mort, ce n'est rien. Ça existe - mais ce n'est rien ${ }^{15}$ ». Barabbas le quitte donc précipitamment, sortant « dans les ténèbres de la nuit ».

Il se détourne alors des disciples, ne comprenant rien à leurs mystères, à celui de la souffrance et de la mort.

Lorsque ceux-ci apprennent qui il est vraiment, Barabbas den frikände, ils ne peuvent s'empêcher d'un mouvement de recul, d'horreur même. C'est donc l'homme à la place duquel le Fils de Dieu est mort. Barabbas le libéré devient Barabbas l'exclu.

Mais son seul vrai désir est, au fond, de rester ce qu'il a toujours été - «vara sig själv och inget annat ${ }^{16} »$. Un désir vain dorénavant.

Bientôt Barabbas assiste au début des persécutions, à la lapidation du Bec-de-lièvre qu'il ne peut, ou qu'il n'ose empêcher, même s'il poignarde à la dérobée celui qui a lancé la première pierre.

60 En cachette il emporte le corps de la martyre pour aller l'ensevelir à côté de celui de son enfant, l'enfant qu'elle avait eu jadis de... Barabbas lui-même.

61 Est-ce un acte d'amour qu'il accomplit là ?

Ensevelir les morts, cela fait bien partie des préceptes d'amour chrétiens. Mais Barabbas a d'autres motivations encore. C'est son passé qu'il enterre.

Le Bec-de-lièvre a su rendre témoignage, dans l'extase, comme son Maître le lui avait demandé et comme elle l'avait ardemment désiré. Mais où était-il resté, son Maître ? Où 
était-il resté son Sauveur, se dit Barabbas, qui sent à cet instant précis combien il le hait, ce Sauveur et ce Maître.

La prostituée chez laquelle Barabbas retourne ensuite, constate avec dépit qu'il n'est plus le même, et elle se demande s'il n'est pas possédé par un esprit, peut-être l'esprit de ce rabbi mort qui cherche à revenir. Barabbas a perdu son identité. Lui, le libéré, il est, en fait, dépossédé de sa liberté.

Ce processus, celui de la Entfremdung, qui a commencé près de la Croix, se poursuit inexorablement.

Les chemins de la liberté, c'est cela pour Barabbas.

, dont il avait été le chef et revient dans la montagne, ne le reconnaissent plus. Il reste des journées entières, rêveur et le regard vide, chef devenu inutile et incapable. Puis, un jour, il disparait dans le désert.

L'histoire de Barabbas aurait très bien pu s'arrêter là, profitant ainsi d'une remarquable unité de temps et de lieu ${ }^{17}$.

Mais Pär Lagerkvist, s'entourant à peine de quelques précautions d'ordre oratoire, simples artifices de style, et sans chercher des justifications d'ordre historique, impossibles d'ailleurs, nous rapporte, dit-il, quelques rumeurs, des on dit à propos du destin de Barabbas.

Puis il enchaîne rapidement, avec fermeté :

Mais ce que l'on sait avec certitude, c'est que vers l'âge de cinquante ans passé il arriva comme esclave dans le palais du gouverneur romain de Paphos, après avoir passé quelques années dans les mines de cuivre de Chypre $^{18}$.

Et la description, le cours de l'histoire reprennent. Le libéré est devenu esclave, un homme aux cheveux gris.

C'est la deuxième étape de son calvaire à lui, de sa descente aux enfers, au sens littéral du terme, car la vie dans les mines de cuivre est un véritable enfer.

Barabbas y est enchaîné à un autre esclave, Sahak, un Arménien, qui forme avec lui un couple étrange. Il est comme embrasé d'un feu intérieur, car il est disciple du Christ. Et lorsqu'il apprend, par bribes, la véritable identité de Barabbas, il en éprouve une joie et une gratitude profondes. Il est donc enchaîné à quelqu'un qui a vu Dieu. Pris d'une curiosité impatiente, il lui pose question sur question à propos des événements de Jérusalem. Des questions qui sont toutes inspirées par la rhétorique des sectes religieuses, sur la venue du Royaume et la nécessité de la mort du Sauveur.

Mais Barabbas lui répond de façon évasive, sauf pour les ténèbres du Golgotha: les ténèbres, ça, il les avait bien vus !

Sahak lui confie alors un secret. Il porte, gravé sur le revers de sa plaque d'esclave, le signe du Christ, pour montrer qu'il lui appartient et qu'il croit en lui.

Barabbas lui demande de graver le même signe sur sa propre plaque. Lui aussi, il voudrait bien croire, être disciple.

Signe d'appartenance, signe d'élection, signe fatal!

Et soudain ils tombèrent à genoux tous les deux, dit le texte, dans une ardente prière à leur Maître, le Sauveur et le Dieu de tous les opprimés ${ }^{19}$.

Les voyant ainsi, oisifs apparemment, le surveillant se jette sur eux et les fouette jusqu'au sang. C'était la première fois, ajoute l'auteur, que Barabbas souffrait pour le 
Crucifié, pour ce rabbi au visage pâle et à la poitrine glabre qui était entré dans la mort à sa place.

Mais tout cela, le rabbi et sa doctrine d'amour, la prière, cela reste un mystère pour Barabbas, et il ne priera plus. Bien que, sans doute, il l'aurait voulu encore. Mais il ne peut pas.

Sahak est, en quelque sorte, l'utopie de Barabbas, celui qu'il ne sera jamais, mais qu'il aurait voulu être, le croyant, celui qui a vaincu le doute.

Revenus à la surface de la terre après bien des années, à la faveur insigne d'un surveillant qui s'intéresse au Dieu de Sahak, ils seront bientôt dénoncés aux autorités par un borgne auquel Sahak avait expliqué que son Dieu viendra pour libérer tous les esclaves. Ils sont donc traînés devant le procurateur par ce borgne, quelqu'un qui ne voit, symboliquement, que la moitié de la réalité, car leur croyance représente un danger social.

2 Sahak, dont l'interrogatoire constitue, pour le lecteur, et objectivement, une réponse, simple, sans équivoques, à toutes les questions que Barabbas se pose depuis le début, confesse sa foi devant le tribunal. Il est condamné à la crucifixion, alors que son compagnon, secouant la tête dans un geste de désespérance, répond au procurateur, d'une voix presque inaudible et sans lever la tête : «Je n'ai pas de dieu ». L'inscription «Christos Jesus » sur sa plaque n'est qu'un malentendu, «parce que j'aurais bien voulu croire ", ajoute-t-il. Et un soldat la barre d'un coup de stylet énergique.

3 Barabbas, le sans Dieu, qui n'a d'autres attaches désormais que ses chaînes d'esclaves, dont le signe d'appartenance vient d'être barré sur la plaque, est donc épargné. Epargné une seconde fois!

Comme dans un état second - depuis longtemps il n'est plus lui-même - il assiste à la mort de Sahak sur la croix.

Cette scène se déroule "dans l'indifférence des hommes et de la nature ", contrairement à ce qui s'était passé lors de la mort du Christ, avec laquelle elle constitue un parallèle. Et pourtant elle est d'une intensité dramatique tout à fait pathétique.

8 Pour les soldats c'est une affaire de routine. Et pour Barabbas, le seul spectateur, caché derrière les buissons, une épreuve insupportable. Lorsqu'il voit mourir son ami, lorsqu'il comprend que c'est fini, "sans que cette fois les ténèbres ne viennent recouvrir la terre », il tombe à genoux, comme s'il priait. « Mais il ne priait évidemment pas, car il n'avait personne à qui adresser sa prière ${ }^{20}$ ".

Barabbas le libéré, Barabbas l'esclave, Barabbas le sans Dieu touche ainsi au fond de sa solitude existentielle. Et cachant son visage barbu dans ses mains, il se met à pleurer.

Les larmes de la déréliction, de la perte de soi $^{21}$.

Le drame de l'abandon total est consommé.

Mais ce n'est pas pour autant le point final de la trajectoire de Barabbas. Pär Lagerkvist le réintroduit dans le monde, par la grâce du gouverneur qui l'emmène dans sa suite lorsqu'il retourne à Rome.

Nouvelle espérance de salut, comme au quatrième acte d'un drame classique, lorsque tout semblait déjà perdu? 

sa condition. que de morts. c'est donc cela. monde. arrivé ! ».

En fait, cette troisième étape, dans laquelle l'auteur entraîne son personnage, sera celle de la fausse chance et du malentendu final, celle de la vanité de toute rébellion contre

Les splendeurs de Rome n'apparaissent à Barabbas que comme à travers un brouillard. Il ne s'en soucie, ni s'en émeut. Ses yeux, dit l'auteur, ne reflétaient plus rien du tout, ils étaient peut-être trop profondément enfoncés dans leur orbite. Il n'est plus qu'un hôte dans cette réalité-là.

4 Mais son indifférence n'est qu'apparence. Car le monde, il le hait. Un jour, il entre, comme par méprise, dans le temple d'Isis. Mais le gardien, devenu méfiant, le chasse. Devant la porte du temple, Barabbas se met à faire des signes secrets et mystérieux, comme pour se protéger. Mais de quoi ? Sa plaque d'esclave avec l'inscription barrée le brûle comme un feu. Il rêve qu'il est enchaîné à un autre esclave, mais il n'est plus enchaîné à personne. A personne au monde.

Retrouvant un jour le signe de Sahak sur les murs de Rome, il devient attentif. Surtout lorsqu'il entend deux autres esclaves dire qu'ils vont participer à des «agapes fraternelles " sur la Via Appia. Les suivant de loin, il se faufile dans les galeries souterraines des catacombes, où il se perd et où il a l'impression de ne plus être entouré

Le royaume de la mort !

Pris de panique, il s'enfuit.

Mais dehors il fait nuit noire, là aussi, et il comprend qu'il ne peut en être autrement, car il est enfermé, emmuré en lui-même - instängd i sig själv. Le royaume des morts,

La mort ! Il l'avait en lui-même, il l'avait eu en lui depuis le début. Elle le ramenait

de force dans le dédale de sa propre existence, le remplissait de sa terreur ${ }^{22}$.

Pour en sortir, s'interroge-t-il, faut-il faire comme les autres, avec leurs agapes fraternelles? Älsken varandra!

Les autres, où sont-ils d'ailleurs, se dit Barabbas, en se trainant, seul, dans les ruelles.

Quand soudain il sent l'odeur de la fumée, voit les premières flammes surgir et entend les cris qui s'élèvent de toutes parts : «Ce sont les chrétiens! ».

Il comprend. Maintenant il comprend !

Ce sont bien les chrétiens qui mettent le feu à ce monde d'horreur.

Leur heure est venue, leur Sauveur est là.

Et dans une sorte d'exaltation schizophrénique qui s'empare soudain de lui, il hurle que cette fois il ne va pas manquer. Le crucifié était de retour, et Barabbas, le frère maudit, n'allait pas lui faire défaut, pas maintenant qu'il avait besoin de lui pour détruire ce

Pris de rage, il fonce à travers les rues et les ruelles, jetant le feu partout. Et quand tout n'est plus qu'un immense brasier, il pousse ce cri de jubilation : « Vois, son Royaume est

Ce cri cependant n'est qu'un hymne au néant, car celui qu'il pense aider, le sauveur qu'il croit venu, c'est le négateur, l'esprit du mal.

8 La peur de la mort qu'il porte en lui a poussé Barabbas, dans une sorte de vertige, à cette dernière et tragique méprise. 
109 À la prison du Capitole, où Néron l'incendiaire a fait jeter les chrétiens, Barabbas se retrouve une dernière fois parmi eux, à l'écart, les écoutant parler de la vie éternelle. La vie éternelle ! Y avait-il seulement un sens à cela ? Pas même à celle qu'il venait de vivre.

111 La parodie de fin du monde mise en scène par Néron, à laquelle il s'était joyeusement prêté, le laisse anéanti. Barabbas le libéré n'est sorti de son indécision, de son doute, que pour se lancer au devant du néant.

112 Lorsque les soldats emmènent les prisonniers pour les crucifier, ceux-ci s'en vont deux par deux. Mais comme ils sont en nombre impair, il reste un dernier. C'est Barabbas, qui marche tout seul, à la fin, ne parlant avec personne. Exclu de la communauté jusqu'à son dernier instant, il est aussi le dernier à mourir sur la croix, lorsque tous les spectateurs sont déjà partis.

Et sentant la mort venir, celle dont il avait toujours eu si peur, il dit à l'obscurité, comme s'il s'adressait à elle : - À toi je remets mon âme !23

Barabbas le libéré, Barabbas l'esclave, Barabbas le sans Dieu, qui s'est débattu avec Celui auquel il n'a pas pu croire, expire, tourné vers ces ténèbres qui étaient son véritable lot.

Le mal d'être a débouché sur rien, sur le non-être.

\section{III}

115 Cette perspective finale du roman est empreinte d'une sombre désespérance, et elle ne doit rien au Schicksalsdrama, malgré ce qu'il pourrait en paraître.

116 Par une ironie suprême Barabbas meurt en martyre d'une foi qu'il n'a jamais eue. Les trois étapes de son errance, dont chacune est marquée par une scène de crucifixion, ont vu sa confrontation avec la personne du Christ, avec la foi au Christ et avec le témoignage pour le Christ. À chaque fois il s'est heurté à son incapacité d'adhérer, donc d'aimer.

117 Si ce personnage de Barabbas a tellement séduit Pär Lagerkvis qu'il a magistralement réussi à en faire un héros de légende, à prêter vie à un nom, c'est qu'il a vu en lui un messager pour l'humanité. Mais un messager sans message précis, comme chez Kafka. Ou plutôt dont le message reste secret, caché, obscur. Indéchiffrable si on ne se réfère pas à l'ensemble de la pensée et de l'évolution de l'auteur.

118 Barabbas, un type plus qu'un individu fortement dessiné, est ce que l'on appelle une âme simple. Notons d'ailleurs que Lagerkvist a toujours montré une prédilection particulière pour les âmes simples. Mais une âme négative.

119 Il ne fait pas partie de ces pauvres en esprit auxquels les Béatitudes ont promis qu'ils verront Dieu. Bien plus, il l'a vu sans le voir, car il reste enfermé en lui-même et ne connaît finalement comme seul moyen d'ouverture aux autres que la haine et la violence. Il est né maudit par sa mère, une femme moabite tombée jadis entre les mains d'une bande de brigands, qui en avait fait son ordinaire et qui s'en était débarrassée lorsqu'il était devenu clair qu'elle était enceinte, et dont le chef Eliahu sera tué bien des années plus tard, dans une lutte singulière, par Barabbas qui lui contestera son autorité sur la bande.

120 Mais ce que l'on ne savait pas, ajoute l'auteur ${ }^{24}$, ce que personne ne pouvait savoir, c'est que cet Eliahu était le propre père de Barabbas. 
Révélation dont l'intérêt dramatique est inexistant dans le contexte, mais que Lagerkvist veut hautement symbolique.

En effet, tous les mouvements de son être portent chez Barabbas la marque de sa révolte contre l'autorité, contre le père. L'auteur, lui prêtant ses propres angoisses, comme si souvent, soulève par son intermédiaire toutes ces graves questions qui ont hanté son esprit depuis le début. La lutte entre le bien et le mal, dont la révolte contre l'autorité est un aspect constant, en est le thème central.

Le premier drame déjà, Människor (Hommes), que Pär Lagerkvist a publié en 1912, met en scène le représentant du mal ${ }^{25}$. Il s'appelle Gustav Mörk (mörk = sombre, obscur), et sa méchanceté est engendrée par son angoisse. Le mal est son destin, en même temps que sa punition, il est en lui.

Par la suite Lagerkvist avait été tenté, pendant sa période de lutte contre le totalitarisme, de considérer le mal d'abord comme une puissance en dehors de l'homme, qui en apparaissait comme la victime désemparée. Comme une "nécessité des temps » aussi, dans Bödeln (Le Bourreau, 1933) par exemple, où c'est un bouc émissaire qui en porte la responsabilité.

Mais il reviendra assez rapidement à la conception première d'un mal irrésistible qui se trouve en l'homme lui-même, à savoir son instinct du mal.

C'est en lui que se résume le mal d'être.

Il en est la racine et la nourriture.

De sorte que la question existentielle du sens de la vie se précise et se réduit à celle du sens du mal. Cette question est au cœur de Barabbas, au cœur d'autres romans encore, comme Dvärgen (Le Nain) paru en 1944.

Le nain est l'incarnation de forces stériles et destructrices, il ne peut aimer et vit dans la haine de tout ce qui est bon et positif. L'action du roman se passe dans le milieu de la Renaissance italienne, avec ses intrigues, ses meurtres, ses fêtes, et le nain, qui est le fou du prince, juge toutes ces vanités humaines. C'est une sorte de Rigoletto négateur, un Mephisto à l'esprit érasmien, personnage tout à fait symbolique qui a "l'expérience des siècles passés dans son regard». Il envie tout ce qui n'est pas comme lui, les recherches esthétiques de l'artiste, l'amour de la jeunesse, les tourments religieux mêmes, qu'il tourne en dérision. Son élément, c'est la guerre, la ruse et la tromperie. Son caractère symbolique du mal apparaît pleinement dans la dernière scène, où il est assis dans la caverne humide de la prison, dans l'attente de son retour dans le monde, de sa « résurrection».

Car le mal, comme le bien, est éternel. Les deux ont une mission métaphysique.

La foi dans la puissance du mal est plus facile cependant, plus évidente que l'autre, celle à laquelle aspire Pär Lagerkvist, et qui constitue le problème de Barabbas.

Celui-ci devine, comme le nain, l'existence du bien. Il ne cherche pas comme ce dernier à le combattre, au contraire, il cherche désespérément à y croire. Ce personnage qui mène une vie d'ombre - ett skuggliv - et qui meurt dans des ténèbres insondables, sent l'emprise étrange que le bien exerce sur lui, en trollmakt dit-il, une emprise qu'il ne sait pas définir et qu'il ne comprend pas, mais qui est telle qu'elle l'oblige à rompre avec le mal, avec son passé criminel. 

intérieure. déchristianisé. sous-jacent ${ }^{27}$. ce, « toi »? Pär Lagerkvist.

Bien que le Christ soit présent dans sa vie, bien qu'il soit capable d'éveiller les sentiments les plus héroïques chez ses partisans, chez Sahak et les autres martyrs, Barabbas ne le comprend pas. Il lui reste inaccessible.

Malfaiteur et fils des ténèbres, il incarne l'inutile et vaine recherche de Dieu, bien qu'il ait connu dans son existence des moments où il était aux portes de la foi et de la paix

Barabbas, tragédie de l'homme prisonnier de sa propre réalité et incapable d'en sortir, qui voudrait bien croire et prier mais qui ne sait ni en quoi ni pourquoi, qui saisit le sens des ténèbres du Golgotha ${ }^{26}$ mais non pas celui de la lumière du matin de Pâques, est l'expression de la méditation métaphysique de Pär Lagerkvist dans un monde

Il n'y a cependant pas de réponse à y trouver, pas de réponse claire aux questions soulevées, peut-être même pas l'amorce d'une réponse.

En effet, la fin pessimiste de Barabas, qui correspond à une sorte de déchaînement apocalyptique, laisse ouvertes les possibilités d'interprétation, malgré le nihilisme

Alors que chez Simone Weil, dont la méditation métaphysique reflète la même problématique du Dieu absent, les mêmes angoisses, penser Dieu c'est assumer l'épreuve du néant, alors que pour elle l'instant de la mort est enfin celui où l'essence de la personnalité d'un être se révèle ${ }^{28}$, Pär Lagerkvist, lui, reste dans une obscurité et, pourrait-on ajouter, dans une honnêteté totales.

Pas la moindre trace même d'un pari pascalien.

Il joue avec son héros, en quelque sorte, le jeu de la relation psychothérapeutique. Son roman est, ainsi vu, un jeu de pistes, de pistes de réflexions psychodynamiques pour des détresses individuelles et universelles depuis longtemps ressenties. Qu'est-ce qui nous condamne à vivre ? À mourir?

La dernière parole de Barabbas - et comment ne pas remarquer ici que c'est quasiment la seule fois qu'il parle dans le roman, portant par ailleurs dans une souffrance muette sa révolte impuissante

- est, en fait, l'expression d'un immense désemparement. Face à l'inconnu, à cette force mystérieuse que sont les ténèbres

- mörkret -, alors qu'il s'écrie "À toi je remets mon âme!", que veut-il dire ? Qui est-

Pour Pär Lagerkvist il y a derrière ce cri bien plus qu'un parallèle facile avec la dernière parole du Christ sur la croix, ou un simple effet de style. Si le Christ s'adresse à son Père, Barabbas, lui, ne s'adresse à personne. Il n'a pas la foi des chrétiens pour qui la mort n'est qu'un passage. Il fait comme si, « comme s'il s'adressait à l'obscurité », et sa mort n'a donc pas de sens.

Pas plus que son dernier cri.

Le mystère demeure, aussi épais que lors de l'entrevue avec Lazare. « La mort, ça existe, mais ce n'est rien ». Le rien, et pourtant pas le néant. Car si c'était le néant, pourquoi lui parler et pourquoi lui remettre son « âme»?

7 Le nihilisme désespéré d'un certain existentialisme n'est donc pas ici le dernier mot de 
Les mêmes problèmes, celui du mal, de la mort, du sens de la vie, seront repris dans les romans suivants, Sibyllan (1956), qui se déroule dans le contexte de la Grèce antique, et Ahasvérus död (La mort d'Ahasvérus, 1960), l'histoire d'un habitant ordinaire de Jérusalem qui, comme Barabbas, a été en contact avec le Christ, et qui est également partagé entre la foi et le doute.

$\mathrm{Au}$ fond d'eux-mêmes Barabbas et Ahasvérus sont fascinés par cet inconnu, ce sont des chercheurs de Dieu. Mais qui sont également maudits parce qu'ils demeurent en marge de l'amour, parce qu'ils ne sont chez eux nulle part, ni dans ce monde ni dans un autre.

Dieu, pour eux, c'est une plaie ouverte, voilà tout.

D’ailleurs, Pär Lagerkvist a plus d'une fois souligné que dans la lutte inégale que se livrent ici le bien et le mal, il n'y a pour lui pas d'instance supérieure, ou divine. Le bien et le mal, cela ne concerne que les hommes, et eux seuls.

Dieu ne s'en soucie pas. Il est ce vieillard sans âge occupé à fendre du bois (comme l'a longtemps fait tout bon grand-père suédois!) que Lagerkvist nous montre dans Det eviga leendet (Le sourire éternel, 1920) et que la foule des morts vient trouver pour lui demander ce que signifie tout ce chambardement cosmique qu'on appelle la vie.

Et Dieu de répondre à peu près ceci : « Euh ... rien de précis. J’ai fait ce que j'ai pu ».

Si les mêmes problèmes sont donc repris par Lagerkvist jusqu'à la fin, et s'il n'y a jamais de véritable solution, de choix bien assumé, et encore moins de certitude, c'est que cet auteur reste un songeur tourmenté, sublime ou terre à terre, qui n'a pas de message à transmettre.

Il ne prend pas parti. Il se drape dans le monde des symboles, où le lecteur peut découvrir, s'il le veut, ses propres réponses à ses propres angoisses.

Et c'est bien là la dernière impression que le lecteur peut retirer de Barabbas. Un roman dont le héros n'est pas vraiment une incarnation du mal, mais plutôt sa victime, portant, symboliquement, le destin de tous les hommes.

$\mathrm{Au}$ temps de l'existentialisme triomphant, lorsque parut le roman, cet aspect a sans doute été celui qui a le plus frappé les contemporains. On a vu en Barabbas un frère ${ }^{29}$, un semblable qui reflétait la position de l'homme moderne pris entre un monde perverti et une foi en un Au-delà devenue problématique.

Les titres par lesquels la presse a salué, déjà en 1950, la sortie du livre, les épithètes employées, en témoignent d'ailleurs abondamment ${ }^{30}$.

Replacé dans le cours de l'Histoire, l'homme moderne a eu le sentiment d'être devant le destin comme un coupable innocent, emmuré dans son Moi et incapable d'aimer, chercheur désespéré d'une vérité qui se heurte à ses propres limites.

60 Mais la recherche d'une vérité amène forcément à rompre ces limites, un jour ou l'autre. "Tu ne me chercherais pas si tu ne m'avais pas déjà trouvé », dit le Dieu de Pascal. Et Simone Weil, reprenant le même paradoxe, pense que l'athée accède à Dieu de par la grâce justement de son refus.

Une recherche de la vérité qui amène ainsi à exorciser le néant tant redouté, et à poser des repères positifs. 


\section{NOTES}

1. - L'essai de H. Söderberg n'avait aucun caractère scientifique et fut très controversé. Même E. Renan, qui avait été un des maîtres à penser de Hjalmar Söderberg, et qui s'était, lui aussi, dans sa Vie de Jésus posé la question de cette supposée homonymie, n'avait osé aller si loin. Söderberg reste, même et surtout ici, ce qu'il ne cesse d'être, un « charmant causeur ».

2. - Pär Lagerkvist a résumé ses impressions dans une série de petits essais intitulée Den knutna näven (Lepoing noué). La terre que Jésus de Nazareth avait foulée de ses pieds, à la fois terre d'ancienne culture et désert aride, lui semblait le symbole même du double déchirement de la condition humaine.

3. - Cf. à ce sujet G. Fredén, Pär Lagerkvist. Från gudstanken tili Barabbas, Stockholm, 1952. Pierre Halleux, Pär Lagerkvist, Bruxelles, 1950. L. Sjöberg, Pär Lagerkvist, New-York, 1976.

4. - Lagerkvist a décrit ce milieu et l'influence qu'il a exercée sur lui dans son fragment autobiographique Gäst hos verkligheten (1925).

5. - Pär Lagerkvist, Gäst hos verkligheten, p. 143.

6. - Ibid.

7. - Parmi les œuvres majeures de Lagerkvist, signalons à côté de Barabbas, Det eviga leendet (Le sourire éternel), 1920, Den osynlige (L'invisible), 1923, Det besegrade livet (La vie vaincue), 1927, Den knutna näven (Le poing noué), 1934, Dvärgen (Le Nain), 1944, Sibyllan (La Sibylle), 1956, Det heliga landet (La Terre Sainte), 1964.

8. - Cf. à ce sujet le Journal de Pär Lagerkvist, 16/04/1951. Kungl. Bibliotheket, Stockholm.

9. - Pär Lagerkvist, Den knutna näven, Stockholm, 1949, p. 105.

10. - Paroles que le Christ adressa à ses disciples.

11. - «Hur en mäimiska ser ut betyder inte mycket», Barabbas, Bonniers, 1972, p. 5.

12. - Cf. à ce propos E. Gibbon, The history ofthe Décline and Fall off the Roman Empire, 1788, réédition New-York, 1946, t. 2, p. 393 ss.

13. - Cette idée a été reprise par Karl Marx, puis par F. Nietzsche (cf. Ch. Andler, Nietzsche, sa vie et sa pensée, Paris, 1931). Ce dernier la manipula avec tant de délices qu'il en est tombé dans le caricatural, notamment dans son Antéchrist et dans La volonté de puissance.

14. - On ne peut s'empêcher de songer à la remarquable interprétation du personnage qu'a donnée Anthony Quinn dans le film tiré du roman de Lagerkvist.

15. - «Dödsriket, det är ingenting. Det finns - men det är ingenting» (Barabbas, Bonniers Aldus, 1972, p. 47).

16. - Ibid., p. 41.

17. - En 1953 Pär Lagerkvist tira de son roman une version dramatique qui fut donnée au Dramaten à Stockholm avec un grand succès.

18. - «Men vad man vet med bestämdhet är att han ett stycke upp i femtiårsåldern kom som slav tili den romerske ståthållarens hus i Paphos efter att ha tillbragt några år i de cypriska koppargruvorna» (Barabbas, op. cit., p. 76).

19. - Barabbas, op. cit., p. 84.

20. - «Men han bad ju i själva verket inte. För han hade ingen att bedja tili» (Barabbas, op.cit., p. 110).

21. - Cf. Heidegger (Etre et Temps, 38) qui voit dans la déréliction une forme de déchéance de l'être-là, du Dasein.

22. - «Döden! Den hade han alitid inom sig, den hade han haft inom sig så länge som han levât. Den jagade honom med sin skräck» (Barabbas, op. cit., p. 119).

23. - «Nar han kände döden komma, den som han alitid hade varit så rädd för, sade han ut i morkret, som om han talade tili det: Till dig överlämnar jag min själ» (Barabbas, op. cit., p., 129). 
24. - Cf. Barabbas, op. cit., p. 69, 72.

25. - C'est un sombre drame, écrit dans un style décadent, mettant en scène la rivalité de deux frères ennemis et imitant les Räuber de Schiller. Lagerkvist était alors à la recherche de l'expression forte. Ce thème du drame entre deux frères incarnant le bien et le mal sera repris en 1939 dans Seger i mörker.

26. - Il est surprenant de voir dans le roman combien les ténèbres sont la seule chose de la Passion dont Barabbas se souvienne vraiment, qui l'inquiète et à laquelle il songe tout le temps.

27. - Cf. JömnMjöberg, Livsproblemet hos P. Lagerkvist, Stockholm, 1951.

28. - Cf. Simone Weil, La connaissance surnaturelle (1949).

29. - Cf. par ex. J. Paillard, Barabbas, min broder, Nya Argus, 1951.

30. - Cf. Aftontidningen, 7.9.50 : «Korset och kedjan» (La Croix et la chaîné), Stockholm Tidningen, 7.9.50: «Martyr utan tro» (Martyre sans foi), Svenska Dagbladet, 7.9.50: «Mannen utan gud» (L'homme sans Dieu), Arbetaren, 14.9.50 : «Barabbas - människan » (Barabbas, l'homme), etc.

\section{RÉSUMÉS}

Barabbas - un nom qui apparaît de façon éphémère dans les Écritures, et qui disparaît tout aussitôt. Mais pour devenir quoi ? Cette question sera le point de départ, et le point d'arrivée, du roman de Pär Lagerkvist, où l'auteur, en suivant la trajectoire supposée de Barabbas le libéré, repose le problème qui est à la source de son mal d'être, du mal d'être que reflète après 1945 la littérature existentialiste, celui des relations entre l'homme et le surnaturel, celui de sa liberté dans la lutte que se livrent le Bien et le Mal. L'homme en proie au déchirement entre la foi et le doute, peut-il être sauvé, peut-il échapper à l'angoisse qui est son héritage ? L'instinct du mal qui est en l'homme est la véritable racine et la nourriture du mal d'être.

Employant le matériel historique avec une totale liberté, Pär Lagerkvist nous présente un Barabbas enfermé en lui-même, qui se débat dans un huis clos sartrien, et dont trois étapes jalonnent le processus inexorable de la totale Entfremdung: son errance dans Jérusalem, côtoyant les premiers disciples, sa vie d'esclave dans les mines de Chypre, à côté de Sahak, esclave chrétien qui essaye en vain de lui faire partager sa foi, son arrivée à Rome enfin, où, se faisant incendiaire et participant malgré lui à la parodie de fin du monde mise en scène par Néron, croyant servir le Maître des chrétiens, il meurt sur la croix, en martyre d'une foi qu'il n'a jamais partagée, remettant son âme aux Ténèbres qui étaient son véritable lot. Le mal d'être n'a débouché sur rien, sur le non-être.

Barabbas est l'expression de la méditation métaphysique de Lagerkvist dans un monde déchristianisé. Malgré le nihilisme sous jacent la fin du roman laisse ouvertes cependant les possibilités d'interprétation. Mais l'impossibilité de croire demeure entière, et Lagerkvist reste, selon sa propre parole, "un croyant sans croyance ", un songeur tourmenté qui n'a pas de message à transmettre, qui se drape dans le monde des symboles, où le lecteur peut trouver, s'il le veut, ses propres réponses à ses propres angoisses.

Barabbas - bloss ein Name, der nur flüchtig in den Evangelien erscheint, und sofort wieder aus dem Feld rückt. In welches Dasein verbannt?

Von dieser Frage geht der Roman von Pär Lagerkvist aus, und auf diese Frage stösst er auch wieder am Ende. Indem er den vermutlichen Lebenslauf von Barabbas dem freigelassenen verfolgt, wirft Lagerkvist das Problem auf, das sich an der Quelle seines Weltschmerzes befindet, 
eines Weltschmerzes der sich in der Literatur des Existentialismus nach 1945 widerspiegelt, das Problem seiner Freiheit inmitten des ständigen Kampfes zwischen Gut und Böse. Kann der zwischen Glauben und Zweifel zerworfene Mensch gerettet werden? Kann er sich von der Daseinsangst, die sein Los ist, befreien? Das Böse in uns, meint Lagerkvist, ist die Wurzel des Weltschmerzes und seine Nahrung.

Indem er mit dem historischen Material ganz frei umgeht, führt uns Pär Lagerkvist einen in seinem eigenen Ich eingeschlossenen Barabbas vor, dessenWeg zur letzten, sinnlosen Vereinsamung im Nichts über drei Stationen führt: sein Umherirren in Jerusalem, in der Nähe der ersten Jünger, sein Leben als Sklave in den Kupferminen Zyperns, neben dem Christen Sahak, der vergebens versucht, ihn zu seinemGlauben zu bekehren, seine Ankunft schliesslich in Rom, wo er sich als Brandstifter an dem von Nero in Szene gesetzten Ende der Welt beteiligt, aus Versehen, da er meint dem Herrn der Christen zu dienen, und als Märtyrer eines Glaubens, den er nie besass, am Kreuze stirbt, seine Seele der Finsternis, die immer sein Los blieb, anvertrauend. Der Weltschmerz mündet so ins Leere, ins Nicht-Sein.

Barabbas ist der Ausdruck von Lagerkvists metaphysischer Suche in einer dem Christentum entfremdeten Welt. Trotz seines spürbaren Nihilismus lässt der Roman verschiedene Möglichkeiten der Interpretierung offen. Aber am Ende bleibt der Glauben weiterhin eine vollkommene Unmöglichkeit. Lagerkvist steht da, nach seinem eigenen Wort, als «ein glaubender Mensch ohne Glauben», ein Grübler, der keine Botschaft zu übermitteln hat, der sich in die Welt seiner Symbole verhüllt, wo der Leser, wenn er es will, die eigenen Antworten auf seine eigenen Seelenängste finden kann.

\section{AUTEUR}

\section{GEORGES UEBERSCHLAG}

Université Charles de Gaulle, Lille III 